\title{
Promoting "6s" Management Principle and Realizing Lean Management in Large-scale Technical Transformation Field Safety
}

\author{
Yuan Dan \\ Dalian Power Supply Company of National Grid Liaoning Electric Power Co., Ltd. \\ Henxue1997@163.com
}

Keywords: $6 s$ construction site controllable controllability

\begin{abstract}
As the basic activity of production site management, "6s" management focuses on the improvement of working site arrangement, adjustment, clean-up, quality and safety. It is in fact to make a comprehensive consideration over the environment of production site and implement practical measures or standardized management on the production site to ensure a clean, beautiful, neat and standardized working environment, so as to ensure the good mental outlook of employees and safe production quality. Dalian Power Supply Company combines "6s" management principle with the practical safe working environment and implements " $6 \mathrm{~s}$ " management over the entire large-scale site renovation project of substation, realizing the objectives of "zero error" in the switching operation during project construction, "zero peccancy" of workers and "zero defect" of equipment operation, promoting the improvement of safety management level of working site and quality of the personnel and truly achieving the "controllable, controllability, in control" construction site.
\end{abstract}

\section{Description of the Management Objectives}

\section{Management Concepts and Strategies}

1) Management Concepts

Promote safety self-management, clearly define responsibilities and obligations of all levels of personnel in the "6s" management, improve employees' safety awareness and guide the staff to pay attention to safety actively. The personnel are classified into three levels: team, grass-roots unit and the company. "6s" management of the team focuses on "people", the concept of "people first" runs through the whole management process and improvement of safe production motivation and innovation of all workers is taken as the main line. "6s" management of grass-roots unit keeps a foothold on the "norms" and "assessment" to standardize the procedures of daily safe production and strictly examine signs and risks of habitual peccancies. The company implements the advanced safety management mode of "6s" management actively and puts high efforts to promote PDCA (Plan - Do - Check - Action) management mode; the company takes its first step in establishing a scientific and complete safety responsibility system, which takes administrative chiefs as the core and the first responsibility person of safety, and takes deputies as the first responsibility person of safety for the cantonal area; the company takes safety objectives and work as priorities and establishes a "three-dimensional safety net" to conduct "6s" management gradually and orderly.

2) Management Strategies

(1) Obtaining Supports from the High Level

Supports from the high level are one of most important issues for the carrying out of " $6 \mathrm{~s}$ " activities. Especially for that "6s" management mode may not adapt to the production site in the very beginning, supports from the leaders are the guarantee for the smooth conduction of activities.

(2) Establishing "6s" Promotion Organization

In order to better implement "6s" management, the company cannot just dispatch several staff to finish the heavy workload. Instead, the company should establish a "6s" promotion organization 
controlled by selected staff to conduct activities, which is the key to the successful implementation of "6s" management.

(3) Improving “6s” Inspection Standards

For on-site staff, production is the core work and it would not be feasible for them to carry out "6s" activities without providing standards. Therefore, the standards of inspection should be designed and completed before the carryout of activities. Various fun activities can be adopted to improve the sense of participation and every employee can be encouraged to propose ideas actively.

(4) Designing a Reasonable Appraisal Plan

"6s" activities can be regarded as cultural activities of the company in the appraisal form, which can encourage all departments to be get involved and do well. Therefore, it is necessary to design a reasonable appraisal plan.

Management Index System and the Target Value

1) Index System

(1) Accidents with deaths in safety production

(2) Accidents with deaths of site workers in award contract, contract and subcontract projects

(3) Accidents with severe injuries in production

(4) Malignant operational accidents at or above $10 \mathrm{kV}$

(5) Large or above grid or equipment accidents with responsible person

(6) Pass rate of work tickets

(7) Pass rate of operating tickets

2) Indicators Decomposition and Target Value

The company defines clearly the target value of all specialties and units and understands the indicators.

\section{Main Management Practices}

\section{Human Resources to Ensure the Normal Operation of Procedures}

To ensure the safe, orderly and high-quality construction of renovation project on the $66 \mathrm{kV}$ power distribution unit of Wutun substation, the company establishes a leading group and site command center for this project. The leading group is in the charge of deputy general production manager, responsible for the leading and coordinating work of the project. Deputy is assigned to deputy chief engineer and the members are director of Department of operation and maintenance, security quality department, substation operation and maintenance department and electrical construction companies, etc..

Site command center is headed by the deputy chief engineer and team members are directors and representatives of Department of operation and maintenance, security quality department, substation operation and maintenance department and electrical construction companies, etc.. Members of site command center remains at the field through the construction process to coordinate and solve all problems during the construction and ensure the safe, orderly, high-quality completion of the project.

\section{Performance Evaluation and Control of Professional Management to Ensure Normal Operation of Procedures}

In accordance with the responsibilities of various departments and specialties and construction units, the responsible employees should be punished if there is any discovery of problems on the site according to the company safety regulations and punishment regulations on site work peccancies of provincial company. Rewarding and punishments are both heavy in the site safety management and the site production safety managing staff should be punished at the highest level if they fail to discover or stop unqualified site operations and be rewarded accordingly if they finish the safe production goals.

1) Performance Assessment Organizations 
They are department of safety supervision and human resources department in the company.

2) Performance Appraisal Index System

(1) No accident with deaths in safety production

(2) No accident with deaths of site workers in award contract, contract and subcontract projects

(3) No accident with severe injuries in production

(4) No malignant operational accident at or above $10 \mathrm{kV}$

(5) No large or above grid or equipment accident with responsible person

(6) $100 \%$ pass rate of work tickets

(7) $100 \%$ pass rate of operating tickets

3) Performance Evaluation Process and Description

Rewards and punishment scheme should be proposed by the department of safety supervision and implemented after being checked by the human resources department and approved by the company leaders.

The effective performance evaluation system is beneficial for the completion of site construction safety work management, clean-up of the construction site environment and avoidance of peccancies on management, operation and labor discipline. The company truly implements the three irons to prevent three peccancies and also realizes heavy rewards for those careful and responsible ones and punishment for those with peccant behaviors, achieving fairness among construction workers and encouraging them to inspect themselves' defects and make summaries, so as to improve the safe production management level and achieve the win-win objective.

\section{Evaluation and Improvement}

\section{Professional Evaluation Methods}

The application of "6s" management to the large-scale reconstruction project of transformers is a breakthrough of the traditional model of safe production and management. The seamless joint of each department can timely and effectively handle various puzzling questions, thus substantially enhancing the quality of safe production and working efficiency, ensuring the safe and orderly construction transformation and advancing the safe operation of power grid.

Through the evaluation of 6S management, the company analyzes the hidden dangers and questions in the practical work and extensively seeks opinions so as to take proper actions and improve the applications.

\section{Issues in Professional Management}

1) Imbalanced Safe Operation in Construction Unit. Workers from the advanced units will take the initiative in their works and proactively engaged in various activities. At the same time, innovative measures and complete institutions are formulated for safety and risk management and control. With high enthusiasm, the employees take the lead in all works of the company; however, few of the units are lack of the innovative ability and measures for safety and risk management and control and plagued by insufficient propaganda work. Such fact has demonstrated the huge gap between the safe management level among construction units.

2) Power grid construction features daunting tasks, multiple transient mode, concentrated completed projects, difficult operational control and increasing potential safety hazards, which have reduced the reliability of power grid during the construction. The equipment operation and maintenance units fail to pay sufficient attention to the dangers, or exercise strict supervision and inspection of the construction units.

3) The lean and standardized degree of safe production and management cannot suit the requirements for rapid development of power grid; after the changes in production and operation, the relevant standards should be improved and standard strictly exercised.

4) Some construction enterprises are plagued by weak safety management; some are even engaged in brutal construction as well as operation and command against rules; the large-scale 
concentrated operation will result in the decreasing quality of some of the new equipment and pose enormous pressure to production, coordination, acceptance and operation of power grid.

\section{Future Improvement Orientation and Countermeasures}

1) Strengthen the 6S Educational Training for Safety Management and Deepen the Building of Company Safety Culture

It is demanding to proactively explore the new thoughts for educational training of $6 \mathrm{~S}$ safety, strengthen the job qualifications, safety qualities and risk identification abilities of the managers and field workers, formulate and put into practice the training plan and responsibility. By holding competitions in production and post businesses, the relevant department should provide a platform for the employees to display their proficiency and skill so as to further stimulate and mobilize the enthusiasm of the employees of learning skills, diligently studying their professions, enhancing their job qualifications and increasing the safety qualities and risk identification ability. Meanwhile, it is necessary to strengthen the safety culture building, vigorously publicize the $6 \mathrm{~S}$ safety concept and safety core values and advance the building of corporate safety culture and establishment of long-term mechanism by carrying out anti-violation of regulations and various labor competition activities

2) Set up the Whole-process Management of 6S Safe Production

It is of prime importance to comprehensively carry out the responsibility of the first person responsible for safe production of all levels, enhance the $6 \mathrm{~S}$ quality of managers of all levels, give play to the role of each department and perform the responsibility of safe production in an all-round manner; to continuously improve, forcibly execute and effective supervise the various rules and regulations of safe production; to ensure that everyone should contribute their due share in safe production in order to resolve the practical issues.

Meanwhile, attention should be paid to strengthening of 6S safe management, emphasis of whole-process safe management and whole-process investigation of planing design, purchasing by invitation to bid, equipment surveillance and fabrication, leave-factory check and acceptance, construction and installation, check and acceptance, operation and overhaul and educational training.

These effective safe management can consolidate the basis for the safe management of the company. By September 30, 2014, Dalian company has sustained 2649 days of safe production, hitting a record high.

\section{References}

[1] Chen Shiqing. On the Condition-based Maintenance of Power Transformer [J]. Transformer.

[2] Lu Jizhi. On the Condition-based Maintenance and Development of Electric Transmission and Transformation Equipment[J ].Yunan Electric Power.

[3] Ma Jie. Carry out Equipment Condition-based Maintenance and Ensure the Safe Operation of Power Grid[J].Sci-Tech Information Development \& Economy.

[4] China Planning Press. Collaboration for the Construction and Acceptance Standard of Installation Project of Electrical Device [M].Beijing:China Planning Press.

[5] Chemistry Standardization for Power Plant Technical Committee of Ministry of Electric Power Industry. Collaboration for the Standards of Quality and Test Method of Oil Used for Electric Power [M]. China Standard Press. 